\title{
SRTD-Manet: Secured Routing and Terrific Data Delivery in Mobile Ad-Hoc Networks
}

\author{
C. Lavanya Prabha B.Tech., M.E. ${ }^{1}$, K. Anitha, BE., ME., ${ }^{2}$ \\ CSI College of Engineering, The Nilgiris ${ }^{1}$ \\ Assistant Professor, CSE Dept, CSI College of Engineering, The Nilgiris ${ }^{2}$
}

\begin{abstract}
Mobile Ad-Hoc Networks presume that nodes spontaneous cooperate in order to work correctly. This collaboration process is based on performance and quality.And then some nodes can rubbish to this performance, finding to effecient node processing. Thus, the whole network process could be affected. The use of previous framework is well to find effective nodes. However in this process more energy used for detecting process and lack of network lifetime, to overcome this above problem, we propose a new method based on a secret parameterallocation for major precaution in all type of Networks. In Mobile Ad-Hoc Networks, the estimation load and complication for key management is mainly subject to limitation of the node's accessible resources and the aggressive personality of network. In this proposed work, we present an efficient and secure key management framework (ESKM) for Mobile Ad-Hoc Networks that builds keys by applying a classified dissemination technique and an elemental multicast cluster group. In ESKM, the cluster group creates a view of the certification less key and provides key update process for all nodes, including the clusters themselves.
\end{abstract}

Keywords: Mobile Ad-Hoc Networks, Clustering, efficient and secure key management, multicast routing, data sharing.

\section{INTRODUCTION}

A Mobile Ad-Hoc Networks is a network designed by a huge amount of nodes, each armed with nodes to detetmine natural phenomena such as temperature, light, motion, or sound. The network is manufactured by a "nodes" from a sporadic to some hundreds or equal thousands, wherever each node is attached to one sensor. A Mobile Ad-Hoc Networks node is also known as mote, it is commonly providing with one or number of nodes to get data about the near by coverage area. The different nodes to use, Mobile Ad-Hoc Networks can be executed to support many applications composed with security, entertainment, military sensing and tracking, patient status monitoring, process automation, industrial monitoring, traffic flow monitoring, public utilities, and asset management and Internet of things. Though, many Mobile Ad-Hoc Networks devices have simple source constraints in terms of energy, threshold, calculation, and memory, produced by a requirement to limit the cost of the large number of devices essential for various applications and by settings that avoid easy admittance to the devices. In order to in previous process dynamically give each node validation and establish a pair wise key between nodes, we used key management by using a un paired certificate less hybrid signcryption theme (CL-HSC) planned by America in AN earlier work [10], [11]. CL-EKM is scalable just in case of additives of new nodes once network preparation. CL-EKM is secure against node compromise, biological research and impersonation, and ensures forward and backward secrecy. For overcome this limitations we move on new technique.In this paper, we present a efficient and secure key management framework (ESKM) for Mobile Ad-Hoc Networks. ESKM builds PKI by applying a secret sharing scheme and an underlying multicast server group. In ESKM, the server group creates a view of the certification less authority (CA) and provides key update service for all nodes, including the head nodes itself. We propose an un authorized process to improve key revocation method. The proposed method overcomes the previous problems such as time duration, throughput and energy usage ratio of Mobile Ad-Hoc Networks. We use Network Simulator as a simulator to perform the current method. part II explain the background information about the key management schemes. part III discuss about the new proposed method. Finally simulation and results are discussed in part IV.

\section{RELATED WORK}

According to the secure communication demand in wireless sensor network (WSN),varieties of key foundations are needed. One is pair wise key foundation; the opposite is cluster key foundation. A few schemes has been projected that incorporates 3phase normally [10]:(1) key setup before deployment, (2) shared-key discovery once construction, and (3) path-key foundation if 2 nodes don't share an on the spot key. The most in style pair wise key pre-distribution 
answer is Random Pair wise Key theme [11] which addresses unessential storage drawback and provides some key flexibility. It's supported Erodes and Reni's [9] work. Every sensing element node stores a random set of Nape pairwise keys to target chance $p$ that 2 nodes are connected. Neighbouring nodes will tell if they share a common pair-wise key once they send and receive-"Key Discovering" Message inside radio range. Its defect is that it sacrifices key property to contract the storage usage. Closest (location-based) pair-wise keys pre-distribution theme [8] is another to Random pair wise key strategy. It takes benefit of the situation data to enhance the key connectivity. Later on, Random key-chain based most of the key pre-distribution result is another random key pre-distribution solution that originated from the answer of basic probabilistic of key reconstract scheme [9]. It depends on probabilistic. There are many key reinforcement greetings to build up security of the established link keys, and improve resilience. Objective is to firmly generate a fresh link or path key by using established keys, so the secret's not com- secure once one or a lot of sensing element node is recorded. One method is to extend quantity of key overlap needed in shared key discovery phase. Qcomposite randomly generated key pre distribution theme [11] needs letter common keys to establish a link key. Similar mechanism is projected by Pair-wise key foundation protocol [6] that uses threshold secret sharing for key reinforcement. Chuang et al. [7] and Agawam et al. [8] scheduled a two-layered key management theme and a dynamic key update protocol in dynamic Mobile Ad-Hoc Networks supported the DaffierHellman (DH), severally. However, both schemes don't seem to be fitted to nodes with limited resources and area unit unable to perform valuable computations with massive key sizes (e.g. a minimum of 1024 bit). Since computer code is estimated additional economical and features a short key length (e.g. 160 bit), many approaches with certificate are planned supported computer code. However, since every node should exchange the certificate to determine the pair wise key and verify every other's certificate previously use, the communication and computation overhead increase dramatically. Also, the Base Station (BS) suffers from the upward of certificate management. Furthermore, existing schemes don't seem to be secure. [5] [3].Huang et al. [4] planned a ECC-based key foundation strategy for self-organizing wireless sensor network (WSN). However, we used to give more protection of their theme. Sattam et al. proposed a Certificate less public key cryptography (CL-PKC), this typicaly used to public key cryptography which escapes the essential escrow of identity based cryptography [8]. Hsun Chuang et al. Cooperate with dynamic pair-wise key and cluster key process are shared in more rounds for key material transaction without encryption/decryption and exponentiation processes in Two-layered Dynamic Key Management (TDKM). Nodes (SN) are provided with some degree of properties including energy efficiency, saving capacity, and delay. In academic analysis, Two-layered Dynamic Key Management (TDKM) is correlated with existing key management near display its efficiency.

\section{PROPOSED METHOD}

We proposed an efficient and secure key management scheme (ESKM) for detecting selfish nodes that combines local ombudsman detections and the dissemination of this information on the network.In efficient and secure key management scheme(ESKM), the system public key is distributed to the whole network. The major contributionof our scheme is that efficient and secure key management scheme(ESKM) is designed to provide efficient share updatingamong servers and to quickly respond to certificate less key less updating, which are two major challenges in adistributed process unauthorized scheme.

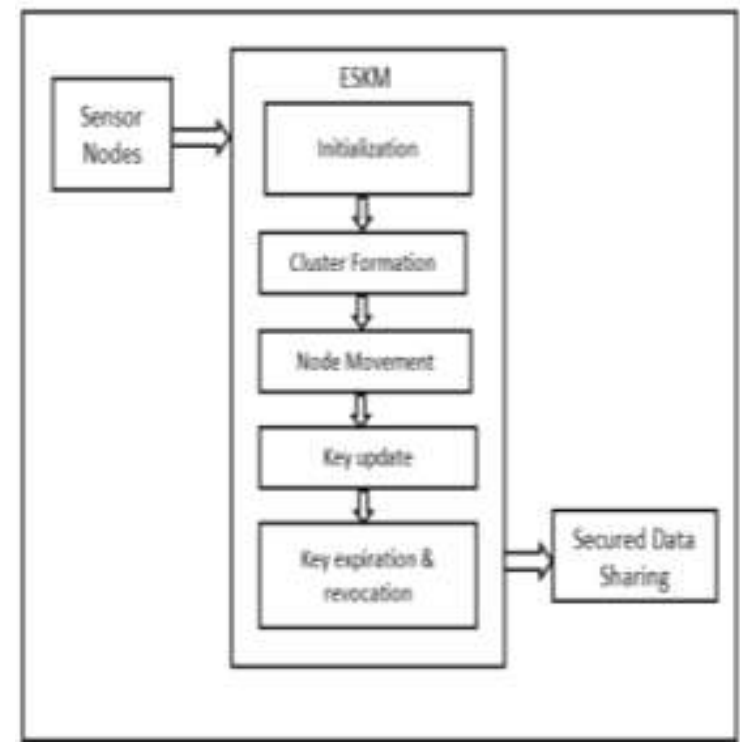

Fig.1. Proposed system process 
The intial technique is that head member form the underlying service group for efficient communication.For efficient, only a pre member of the cluster nodes initiates theshare update phase in eachround. A ticket based scheme isintroduced for efficient certificate less key less updating. Normally, becauseof share updating, recently joining servers could be idle to the system if they mention outdated unauthorized less key less.

Our schemedoes not isolate new servers, and is open for regular nodes foreasy joining and departing. efficient and secure key management scheme (ESKM) creates a view of certification less authority(CA) andprovides secure and efficient service in the mobile and ad hoc environment. In this framework we achieved high performance and accuracy results.This approach reduced time and increases the precision when detecting selfish node..

\section{A. System initialization}

Before the sensor nodes deployment, the Base Station (BS) creates structure parameters and registers the nodes by including it in a member list. The creation of nodes for our proposed key management scheme and sensor nodes are deployed over the region.

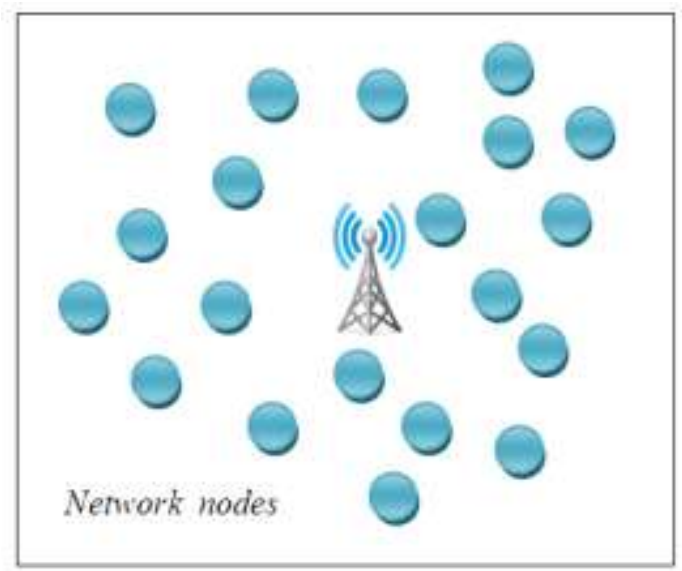

1) Creation of Structure Parameters: The Key Generation Centre (KGC) at the Base Station (BS) runs the following steps by taking a security parameter $\mathrm{k} \in \mathrm{X}+$ as the input, and returns a list of structure parameter.

$\tau=\{F i, E / F i, G, P, P p u b=x P, h 0, h 1, h 2, h 3\}$

2) Node Registration: The Base Station (BS) allocates a unique identifier, denoted by $C a$, to each sensor node $n C a$ and a unique identifier, denoted by $C H b$, to each cluster head $n C H b$,

where $1 \leq a \leq N 1,1 \leq b \leq N 2, N=N 1+N 2$.

During this phase the base station generates a series of parameters using the security parameter $\mathrm{k} \in \mathrm{Z}+$ as the input and returns a list of system parameter $\Omega=\{\mathrm{Fq}, \mathrm{E} / \mathrm{Fq}, \mathrm{Gq}, \mathrm{P}, \mathrm{Ppub}=\mathrm{xP}, \mathrm{h} 0, \mathrm{~h} 1, \mathrm{~h} 2, \mathrm{~h} 3\}$ and $\mathrm{x}$. The BS publishes the value $\Omega$ and keeps the value $\mathrm{x}$ as secret. Then the BS assigns a unique identifier denoted as $\mathrm{Li}$ to each $\mathrm{L}$ node and unique identifier denoted as $\mathrm{Hj}$ to each of the $\mathrm{H}$ node. During this phase, each $\mathrm{L}$ node $\mathrm{nLi}$ chooses a secret value $\mathrm{xLi}$ and computes PLi $=$ xLi P. Then, the BS chooses rLi and then calculates a pair of partial public/private key (RLi , dLi ) described as below:

$\mathrm{RLi}=\mathrm{rLi} \mathrm{P}$

$\mathrm{dLi}=\mathrm{rLi}+\mathrm{x} \cdot \mathrm{h} 0(\mathrm{Li}, \mathrm{RLi}, \mathrm{PLi}) \bmod \mathrm{q}$

Each $\mathrm{Li}$ then sets skLi $=(\mathrm{dLi}, \mathrm{xLi})$ as its full private key and $\mathrm{pkLi}=(\mathrm{PLi}, \mathrm{RLi})$ as its full public key. BS then chooses a uniform random number $\mathrm{x} 0$ and computes

$\mathrm{KLi}$ =HMAC $(\mathrm{x} 0, \mathrm{Li})$. Process occur for all nodes including $\mathrm{H}$ nodes. After the key generation the Bs creates a member list which includes the details of each node and a revocation node list which include details of revoked nodes. The public/private key, $\Omega$, and the individual key are installed in the memory of each of the node. After the network formation one of the node sends an advertisement message to its neighbouring nodes to setup the pairwise key between them. The advertisement message includes its identifier and public key.

Pairwise master key formation : Here a pairwise master key is formed between any two nodes $\mathrm{nA}$ and $\mathrm{nB}$ with unique IDs $\mathrm{A}$ and $\mathrm{B}$. The process starts when $\mathrm{nA}$ receives an advertisement message from $\mathrm{nB}$. Then it executes the following encapsulation process to generate a long-term pairwise master key $\mathrm{KAB}$ and after that the encapsulated key information, $\phi A=(U A, W A)$ is generated. Then it chooses $1 \mathrm{~A} \in \mathrm{Zq}$ and calculates $\mathrm{UA}=1 \mathrm{AP} . \mathrm{TA}=1 \mathrm{~A} \cdot \mathrm{h} 0(\mathrm{~B}, \mathrm{RB}$, $\mathrm{PB}) \mathrm{Ppub}+1 \mathrm{~A} \cdot \mathrm{RB} \bmod \mathrm{q}$ 
$\mathrm{KAB}=\mathrm{h} 1(\mathrm{UA}, \mathrm{TA}, \mathrm{AA} \cdot \mathrm{PB}, \mathrm{B}, \mathrm{PB})$

$\mathrm{h}=\mathrm{h} 2(\mathrm{UA}, \tau \mathrm{A}, \mathrm{TA}, \mathrm{A}, \mathrm{PA}, \mathrm{B}, \mathrm{PB})$

$\mathrm{h}^{\prime}=\mathrm{h} 3(\mathrm{UA}, \tau \mathrm{A}, \mathrm{TA}, \mathrm{A}, \mathrm{PA}, \mathrm{B}, \mathrm{PB})$

$\mathrm{WA}=\mathrm{dA}+1 \mathrm{~A} \cdot \mathrm{h}+\mathrm{xA} \cdot \mathrm{h}$

where $\tau \mathrm{A}$ is a random string and finally output obtained are $\mathrm{KAB}$ and $\phi \mathrm{A}=(\mathrm{UA}, \mathrm{WA})$. Then, $\mathrm{nA}$ sends $\mathrm{A}, \mathrm{pkA}, \tau \mathrm{A}$ and $\phi \mathrm{A}$ to $\mathrm{nB}$ which performs the decapsulation to obtain $\mathrm{KAB}$.

$\mathrm{TA}=\mathrm{dB} \cdot \mathrm{UA}$

$\mathrm{h}=\mathrm{h} 2(\mathrm{UA}, \tau \mathrm{A}, \mathrm{TA}, \mathrm{A}, \mathrm{PA}, \mathrm{B}, \mathrm{PB})$ and

$h^{\prime}=h 3(U A, \tau A, T A, A, P A, B, P B)$.

Finally if $\mathrm{WA} \cdot \mathrm{P}=\mathrm{RA}+\mathrm{h} 0(\mathrm{~A}, \mathrm{RA}, \mathrm{PA}) \cdot \mathrm{Ppub}+\mathrm{h} \cdot \mathrm{UA}+\mathrm{h} ' \cdot \mathrm{PA}$ then output $\mathrm{KAB}=\mathrm{h} 1(\mathrm{UA}, \mathrm{TA}, \mathrm{xB} \cdot \mathrm{UA}, \mathrm{B}, \mathrm{PB})$.

Otherwise output is invalid.

Pairwise encryption key formation: After the formation of pairwise master key KAB between $n A$ and $n B$, nB chooses a random nonce $r \in \mathrm{Zq}$ and calculates $\mathrm{kAB}=\mathrm{HMAC}(\mathrm{KAB}, \mathrm{r})$ and $\mathrm{C} 1=\mathrm{EkAB}(\mathrm{r}, \mathrm{A}, \mathrm{B})$. Then $\mathrm{nB}$ sends $\mathrm{r}$ and $\mathrm{C} 1$ to $\mathrm{nA}$. After receiving $r$ and $\mathrm{C} 1 \mathrm{nA}$ calculates $\mathrm{kAB}=\mathrm{HMAC}(\mathrm{KAB}, \mathrm{r})$ and decrypts $\mathrm{C} 1$.

\section{B. Cluster formation}

Once the nodes are used, all group head through data shares to node. group head to control a group with the approved node and they exchange a normal group key. The cluster head also establishes a pairwise key with each member of the cluster. We also assume that the cluster head is $n C H b$ with $n C a 1 \leq a \leq n$ as clustermembersnCHb. Establishes a cluster key for $O P b$ secure communication in the cluster. The server group structure should be maintained in the entire lifetime of the network.

However, for a mesh structure, there are possible multiple paths between pair of servers. Thus if one link is broken the alternative link could be utilized instead of launching the costly procedure for breakage recovery.

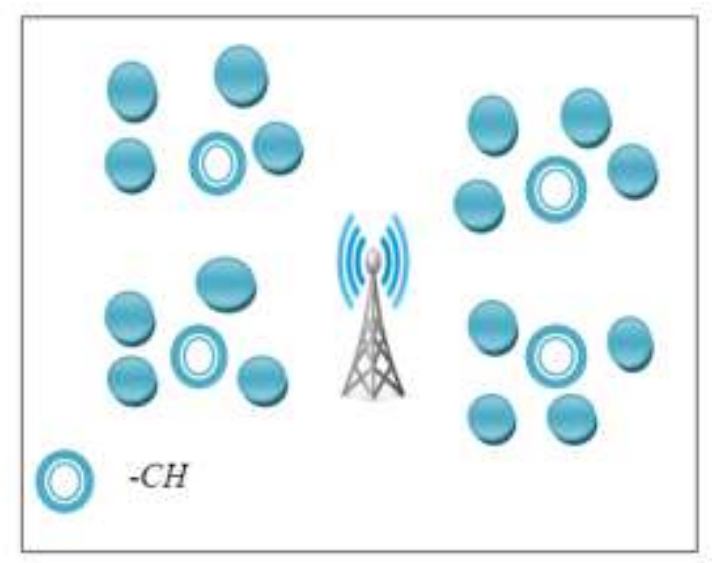

In Efficient and secure key management framework(ESKM), the periodical message Request and Reply are sent out in order to refresh the server group.

\section{Re-Clustering}

In this process re clustering process is used for reduce energy consumption and to increase lifetime and throughput ratio. This re-clustering process is combined more clusters, for example if we having four clusters in a mesh like arrangement it convert to two clusters. Each clusters having more than one cluster heads. Here we combine clusters using bandwidth ratio and frequency range. After re-clustering the cluster key and pairwise key will update to each clusters and nodes. This re-clustering process is combining of clusters. Initially optimum number of clusters for a network is found using the equation

$$
\mathrm{Kopt}=(\sqrt{\mathrm{N}} / \sqrt{ } 2 \pi) * \sqrt{ }(\text { cfs } / \text { gamp }) * \mathrm{M} / \mathrm{d}^{\wedge} 2
$$

Where $\varepsilon f s$ - energy for free space model

Eamp - energy for multipath model

$\mathrm{N}$-no of nodes

The reformation is such that the newly formed clusters have more than one $\mathrm{H}$ sensor. There occurs a switching of header function between the $\mathrm{H}$ nodes using the threshold of time 
$\mathrm{T}(\mathrm{n})=\mathrm{p} /[1-\mathrm{p}(\mathrm{r} \bmod (1 / \mathrm{p}))] \quad$ if $\mathrm{n} \varepsilon \mathrm{G} \ldots \ldots \ldots$ (2)

otherwise

Where $\mathrm{p}=$ no of $\mathrm{H}$ nodes.

$\mathrm{r}=$ current round no. $\mathrm{G}=$ set of $\mathrm{H}$ sensor that is not selected as head.

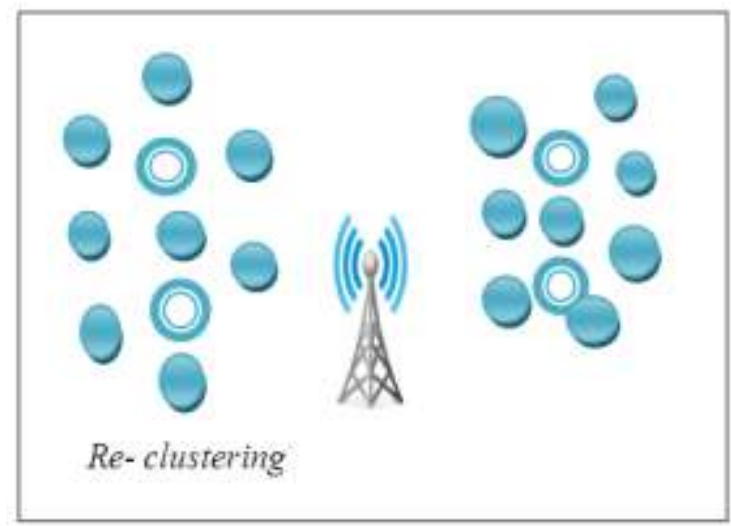

Encryption key updating

Two types of key updating are there and they are pairwise key updating and cluster key updating. There is no need of frequent pairwise master key updating. The pairwise encryption key is updated using pairwise encryption key establishment process. The cluster key updating is done by the cluster heads. Each $\mathrm{Hj}$ chooses $\mathrm{x}^{\prime} \mathrm{j} \in \mathrm{Zq}$ and a new cluster key $\mathrm{GK}^{\prime} \mathbf{j}=\mathrm{HMAC}\left(\mathrm{x}^{\prime} \mathrm{j}, \mathrm{Hj}\right)$ is calculated. Each of the $\mathrm{Hj}$ also generates an update message including HMAC(GK'j,Update) and then calculates C6 = EGKj (GK'j ,HMAC(GK'j ,Update)). After this each Hj transmit the update message and C6 to its members.

\section{Node Movement}

Once a node moves between clusters, the cluster head purely achieved cluster keys to define the forward/backward secretly. Therefore, the cluster head updates the cluster key and informs the Base Station (BS) of the changed node position. Over this report, the Base Station (BS).

1) Forward and Backward secure: Efficient and secure key management framework (ESKM)provides the key update and revocation processes to confirm forward secretly as soon as a node leaves or shared node is identified. Forward Confidentiality is an old key to decrypting the data and Backward confidentiality is a current key from backward encrypting old messages. Forward and Backward Confidentiality are used to secure against node capture attack.

2) Node Leave: A node may leave a group due to node failure, position change or abnormal conversation failure. Herebe located both proactive and reactive ways for the group

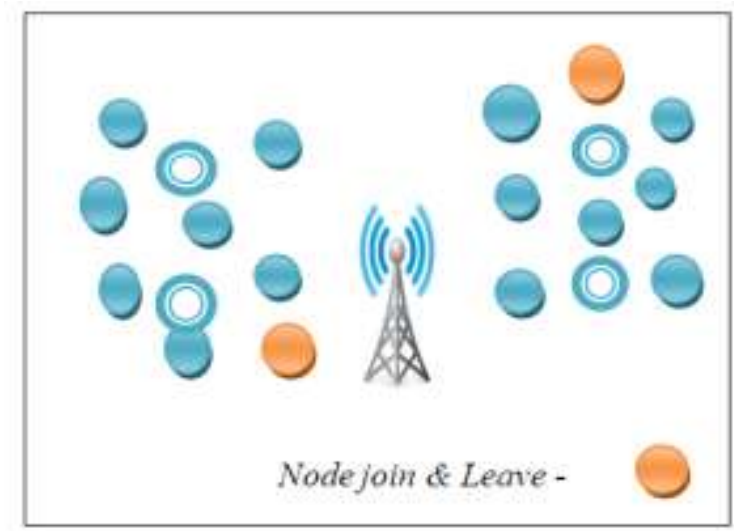

head to find when a node moves the group. The proactive case happens as soon as the nodenCmactively select to avoid the group and send the group head $n C H b$ or the cluster head chooses to revoke the node. Then in this casenCHbcan confirm that the node has left, it transmits a report $E K C H b 0$ (Node Leave, $C m$ ) to update the Base Station (BS) and $n C m$ has left the cluster. When getting the report, the Base Station (BS) is updates the status of $n C m$ in $\mathrm{M}$ and sends a credit to $n C H b$. The reactive case happens when the cluster head $n C H b$ fails to communicate with $\mathrm{nCm}$. It may possibly occur a node expires out of battery power. 
3) Node Join: Once the moving node $n C m$ leaves a cluster, it may join other clusters or return to the previous cluster after some period. We assume that $n L m$ wants to join the athcluster or return to the $b t h$ cluster.

\section{Key Update}

Now in this section we deliver the pairwise key update and cluster key update processes.

1) Pairwise Key Update: Only nodes can update their pairwise key. Toward update a pairwise encryption key, two nodes are to shared the pairwise key perform for in a Pairwise Encryption Key Establishment process.

2) Cluster Key Update: Only cluster head can update their cluster key. If a node attempts to change the cluster key, the node is considered a malicious node.

\section{E. Key Revocation and Addition of a New Node}

We take responsibility that the BS can identify cooperated nodes node and cluster head. The key revocation is nothing but the renewal of keys. The key revocation is calculated by the Certificate revocation list. The Certificate Revocation list split in to two categories given by old certification less authority(CA) and New certification less authority(CA). The Base Station (BS) can require an interference detection system or malicious nodes or adversary's device to detect [13] and [17].

\section{RESULTS AND DISCUSSION}

We use Network simulator version-2 (NS2) to show the performance of our proposed scheme. A wireless ad hoc network consists of 30 nodes are randomly deployed over a square region of $1000 \times 1000 \mathrm{~m} 2$ used in this simulation. The size of the data packet is 512 bytes. Ad hoc on Demand Routing (AODV) protocol is used. We have 2 cluster groups. As compared to existing scheme, our proposed scheme has better performance in terms of energy consumption, Lifetime, and throughput. The following section shows the simulation parameters, results and comparison performance of the proposed system.

\section{Simulation Parameters}

\begin{tabular}{|l|l|}
\hline Parameter & value \\
\hline Field size & $1000 \times 1000 \mathrm{~m}$ \\
\hline Number of nodes & 30 \\
\hline Propagation type & Two ray ground \\
\hline Routing type & AODV \\
\hline Channel & Wireless channel \\
\hline Simulation Time & 85.0 seconds \\
\hline
\end{tabular}

\section{Performance Results}

In this section, the performance of our protocol is compared with the existing method in terms of Lifetime,and throughput.

NetworkLifetime

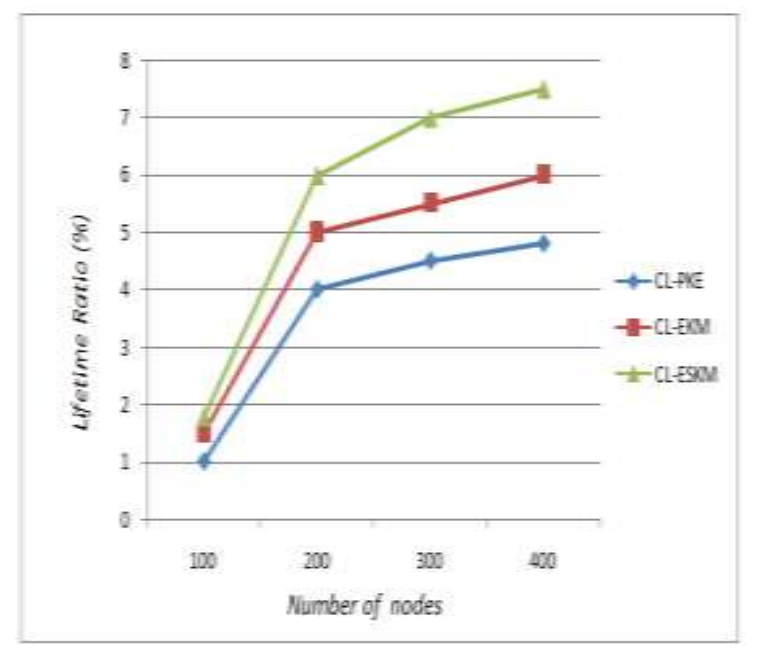


Above graph shows the comparison of existing and proposed key management scheme in terms of Lifetime. In this figure, the performance of proposed key management scheme is increased lifetime ratio level as compared to existing key management scheme.

ThroughputRatio

Bellow graph shows the comparison of existing and proposed key management scheme in terms of Throughput. In this figure, the performance of proposed key management scheme is good Throughput level as compared to existingkey management scheme.

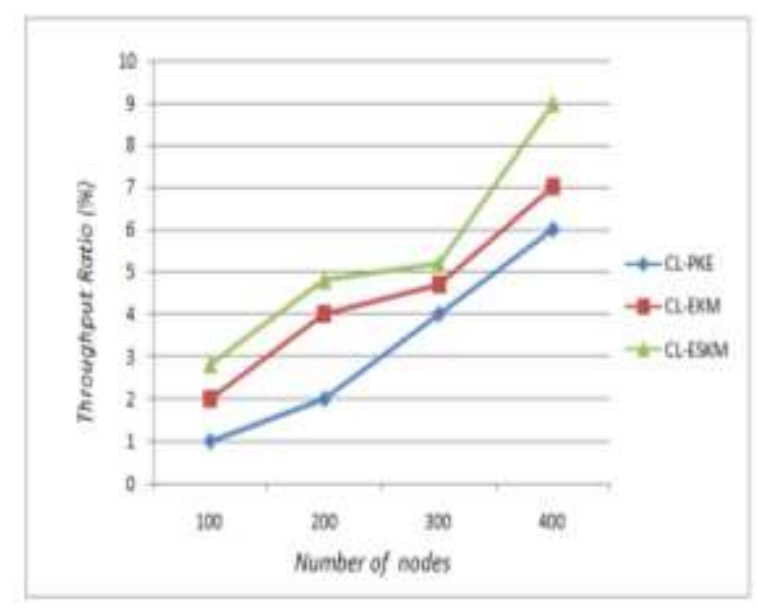

\section{CONCLUSION}

Due to internet connectivity security is an important issue for ad hoc mobile networks. For security we mainly consider the following attributes: availability, privacy, integrity, authentication, authorization and non-denial. Certain security techniques and methods have been construct and present for Ad-hoc network. Key management is the central aspect of the security of Mobile Ad-Hoc Networks, and it is still a pathetic condition. In this paper we propose a new key management scheme, Efficient and Secure Key Management(ESKM) framework, Efficient and Secure Key Management(ESKM)is based on the secret sharing scheme, where the system secret is sharing to a cluster of head nodes. The header cluster creates a view ofcertification less authority(CA). The advantage is that in Efficient and Secure Key Management(ESKM)it is easier for a node to request service from a well maintained group rather than from multiple "self-governing" service providers which may be spread in a whole area. In Efficient and Secure Key Management(ESKM), the server group provides certificate less key less update service for all nodes including the servers themselves. In our future work we will extend Efficient and Secure Key Management Efficient and Secure Key Management(ESKM) to more than cluster heads in large networks and in combined networks.

\section{REFERENCES}

[1]. Akyildiz I.F., Su W., Sankarasubramaniam Y., and Cayirci E., "A Survey on Sensor Network”, IEEECommunication Magazine, vol. 40, no. 8, Aug. 2002, pp. 102-114.

[2]. Alagheband and Aref., "Dynamic and secure key management model for hierarchical heterogeneous networks"

[3]. Carman D. W., Krus P. S, and Matt B. J, Constraints and approaches for distributed sensor network security”. Technical Report 00010, NAI Labs, Network Associates, Inc., Glenwood, MD, 2000.

[4]. Hsun Chuang I., Wei-Tsung Su, ChunYi Wu,Jang-Pong Hsu, Yau-Hwang Kuo.,’'Two-layered Dynamic Key Management inMobile and Long-lived Cluster -based Wireless Networks".,Dept. of Comput. Sci. \& Inf. Eng., National Cheng Kung Univ., Tainan.

[5]. Huang, Q.; Cukier, J.; Kobayashi, H.; Liu, B.; Zhang, J.," Fast Authenticated Key Establishment Protocols for Self-Organizing Networks" TR2003-102 February 2004.

[6]. Jiang P., "A new method for node fault detection in wireless networks," Nodes, vol. 9, no. 2, pp. 1282-1294, 2009.

[7]. Lazos L., and Poovendran R.,. "Serloc: Robust localization for wireless sensor networks".ACM Trans. Sen. Netw., 1(1):73-100, 2005.

[8]. Liu, D. and Ning P. 2003. Establishing pairwise keys in distributed networks. In CCS '03: Proceedings of the 10th ACMconference on Computer and communications security. ACM, New York, NY, USA, 52-61.

[9]. Liu D., and Ning P., "Efficient distribution of key chain commitments for broadcast authentication in distributed sensor networks". In Proceedings of the 10th Annual Network and Distributed System Security Symposium, pages 263-276, 2004

[10]. Paradis L.and Han Q., "A survey of fault management in wireless sensor networks," J. Netw. Syst. Manage., vol. 15, no. 2, pp. 171 -190, 2007.

[11]. Perrig A., Szewczyk R., Tygar J. D., Wen V., and Culler D. E. "Spins: security protocols for networks". Wireless Networking, 8(5):521-534, 2002. 